\title{
CARACTERIZACIÓN PEDAGÓGICA DE LOS ENTORNOS VIRTUALES DE APRENDIZAJE
}

\author{
Pedagogical characterisation of the virtual learning \\ environments
}

\section{Caractérisation pédagogique des environnements virtuels d'apprentissage}

Ángel GARCÍA DEL DUJO y Antonio Víctor MARTÍN GARCÍA

Universidad de Salamanca. Facultad de Educación. Departamento de Teoria

e Historia de la Educación. Paseo de Canalejas, 169. 37008 Salamanca. Correo-e: agd@usal.es,avmg@usal.es

Fecha de aceptación definitiva: marzo de 2003

BIBLID [(1130-3743) 14, 2002, 67-92]

RESUMEN

El trabajo parte de la necesidad de explicar pedagógicamente los llamados entornos virtuales de aprendizaje. Al amparo de los denominados enfoques sociocultural, de la cognición compartida y del aprendizaje situado, así como del paradigma CSCL, el texto trata de profundizar en una línea de reflexión pedagógica sobre las formas como actúan las nuevas tecnologías y, en última instancia, sobre las formas como tienen lugar los procesos formativos en los entornos virtuales y las consecuencias que ello tiene para su diseño y configuración en términos pedagógicos.

Palabras clave: nuevas tecnologías, entornos virtuales de aprendizaje, sistemas de actividad, comunidades de práctica, aprendizaje colaborativo. 
SUMMARY

This study derives from the need to give a pedagogical explanation of the socalled virtual learning environments. Taking into account the socio-cultural approaches of distributed cognition and situated learning, as well as the CSCL paradigm, the text seeks to delve deeper into a line of pedagogical reflection on how the new technologies act, and in the last instance, how the educational processes in virtual environments take place as well as the consequences this has for their design and configuration in pedagogical terms

Key words: new technologies, virtual spaces of learning, activity systems, communities of practice, collaborative learning.

\section{SOMMAIRE}

Cette étude répond au besoin d'expliquer pédagogiquement les nommés environnements virtuels d'apprentissage. Prenant en considération les approches socioculturelles, de la cognition partagée, et de l'apprentissage situé, aussi que le paradigme CSCL, le texte essaie d'approfondir dans la réflexion pédlagogique concernant la façon dont les nouvelles technologies agissent et, au dernier lieu, concernant la façon dont les processus de formation ont lieu dans les environnements virtuels, ainsi que les conséquences que cela a pour sa conception et la configuration en termes pédagogiques.

Mots clef: nouvelles technologies, espaces virtuelles d'apprentissage, systèmes d'activité, communautés de pratique, apprentissage collaboratrice.

\section{Nuevos ESPACIOS DE EDUCACIÓN}

Al final de la década de los setenta y comienzo de los ochenta se quiso ver en lo que por entonces era considerado como nuevas tecnologías, el ordenador, un instrumento cuasi revolucionario aplicado a la educación. Veintitantos años después, las que ahora se autodenominan nuevas tecnologias ${ }^{1}$ siguen presentándose como una herramienta con gran capacidad de transformación de los procesos de enseñanza, aprendizaje y educación. Ni antes ni ahora las cosas son tan evidentes y entonces y ahora las nuevas tecnologías siguen despertando muchas reticencias. Una vez más nos viene a la memoria la certera ilustración de Seymour Papert sobre el ritmo de los cambios en educación (Papert, 1993).

1. El lector pensará inmediatamente en dos cuestiones que, aun siendo de interés conceptual y metodológico, no recibirán por ahora consideración por nuestra parte. Nos referimos, de un lado, a la especificación de las llamadas nuevas tecnologías y, de otro, a las diversas denominaciones que se les atribuye. Somos conscientes, sin embargo, de que en educación éstas no son meramente cuestiones terminológicas, pero no es el momento de detenernos en su análisis. Mayans i Planel.s, J. (2002) Género chat o cómo la etnografía puso un pie en el ciberespacio. Barcelona, Gedisa, 223-243. 
Los profesionales de la enseñanza han mostrado tradicionalmente una actitud de resistencia, hablando entre otras cosas de dificultades técnicas para el uso de estas o aquellas tecnologías, falta de tiempo y formación para una primera valoración en términos pedagógicos, escasa repercusión en las actividades habituales específicamente educativas, dudosa integración en el contexto educativo global... $y$, sobre todo, escasa influencia de la tecnología educativa respecto de la calidad social de la educación.

De las opiniones que estos profesionales dejan traslucir en las conversaciones académicas resultan preocupantes dos tipos de alegaciones: aquellas que se refieren a que el material tecnológico parece carecer de un adecuado tratamiento educativo y enfoque pedagógico y aquellas otras que constantemente aluden a que las nuevas tecnologías no suplantan el ambiente educativo del aula, puesto que no son capaces de recrear adecuadamente esa relación comunicativa entre alumno y profesor; nosotros ampliamos esta segunda interpretación en el sentido de que, según estos profesionales, las nuevas tecnologías no tendrían en cuenta el hálito social, la cualidad social del aprendizaje en el aula, aspecto éste que consideran imprescindible para un buen desarrollo del proceso de enseñanza-aprendizaje y que los profesores hacen pivotar básicamente sobre la relación presencial alumno-profesor.

Y no les falta algún punto de razón; basta con recordar la forma como las nuevas tecnologías han llegado ahora y se han introducido en los recintos escolares, análoga en muchos aspectos a como lo pretendieron hace treinta años, sobre todo en lo que se refiere al desconocimiento o, por lo menos, minusvaloración de las opiniones manifestadas por los profesionales y a la ausencia de reflexión sobre la fundamentación pedagógica que debe soportar la introducción y el uso de las nuevas tecnologías en la educación, dos cuestiones por otra parte estrechamente interrelacionadas.

Precisamente muchas de las percepciones que los profesionales de la enseñanza tienen sobre las nuevas tecnologías dejan entrever o aluden directamente a la ausencia de un marco teórico específicamente pedagógico que interprete adecuadamente no sólo las posibilidades sino también los requisitos que han de cumplirse para que se produzca una relación idónea entre nuevas tecnologías y educación. Nadie pone en duda que buena parte del software que hoy se autocalifica de educativo carece totalmente de fundamentación pedagógica ${ }^{2} \mathrm{y}$, en otras ocasiones, son herramientas apoyadas en unas interpretaciones teóricas manifiestamente insuficientes e incluso erróneas desde una perspectiva educativa.

No son pocos los autores que en este sentido llaman la atención sobre esa especie de determinismo tecnológico que rodea la utilización de las nuevas tecnologías

2. La amplia oferta, que en estos momentos existe en el mercado, de programas de formación en entornos virtuales la hace sospechosa de los propósitos lucrativos por los que se rige; y, al contrario, la educación está tardando en responder a las posibilidades y exigencias de los nuevos escenarios, lo que no resulta menos sospechoso para la pedagogía. Echer erría, J. (2000) Educación y tecnologías telemáticas, Revista Iberoamericana de Educación, 24, http://www campus-oei.org/revista/rie24f.htm. 
en educación y que lleva con frecuencia a un uso indiscriminado de software con independencia de su bondad pedagógica, guiados sólo por las características de la arquitectura técnica que presenta, interpretadas en un sentido bastante próximo al esnobismo, sin reparar siquiera, por ejemplo, en si la topografía que proyecta permite o no algún grado de sociabilidad o si fomenta una visión solipsista de la educación.

Parece como si las nuevas tecnologías, por el simple hecho de serlo y de ser utilizadas, estuviesen llamadas a producir en educación necesariamente transformaciones importantes en cantidad y en calidad, comparables con las que están teniendo lugar, según se dice, en la organización social y económica, en el mundo laboral, en la diversión... y que, sigue diciendo buena parte de la literatura especializada, terminarán por afectar a nuestra forma de pensar y de aprender. Esta interpretación está viéndose reforzada por una lectura de la historia y del desarrollo humano hecha a partir de la tecnología dominante en cada momento para almacenar y transmitir la información (Levinson, 1990; Harnad, 1991; Bosco, 1995; Ong, 1995), aunque no faltan otras visiones más críticas que llaman la atención sobre los múltiples reduccionismos y análisis simplistas ahí implícitos (Olson y Torrance, 1991).

Con todo, cualquier reflexión que tenga en cuenta globalmente unas y otras versiones así como las percepciones que emiten los profesionales de la educación lleva fácilmente a una cuestión que bien podríamos tomar como el punto de partida de nuestro planteamiento: las nuevas tecnologías ya no son en estos momentos, ni deben ser consideradas por más tiempo, simples artefactos o instrumentos que posibilitan la acción o manipulación del entorno por el sujeto. Las nuevas tecnologías son algo más que el elemento o componente material, instrumental, siempre presente en los contextos tradicionales de educación; no negamos que en parte lo seguirán siendo, pero decimos que son algo más que eso.

Las nuevas tecnologías son algo más que meros artefactos o instrumentos, algo más que simples aparatos, otro utensilio más al servicio de un contexto y enfoque tradicional de la educación. Más allá de esta interpretación, vemos en ellas un valor añadido que las convierte en herramientas que generan nuevos espacios para la acción. En este sentido no son simples artefactos, sino artificios capaces de generar nuevos contextos, escenarios diferentes. Son las dos caras, al menos, que presentan las nuevas tecnologías: instrumentos que generan estructuras que posibilitan acciones y viceversa ${ }^{3}$. Y ahí radica la mayor potencialidad y responsabilidad que en

3. Este planteamiento va más allá de la interpretación que hace David H. Jonassen de las tecnologías educativas como herramientas de la mente. Jonassen, D. H. (2002) Computadores como herramientas de la mente, Eduteka, mayo, http://www eduteka.org/ediciones/tema. JonAssen, D. H. \& ReEves, T. C. (1996) Learning with technology: Using Computers as cognitive tools, en JoNASSEN, D. H. (ed.). Handbook of research for educational communications and technology. New York, Macmillan, 693-719. Estamos de acuerdo en que estas tecnologías son algo más que medios de instrucción, pero entendemos que superan también el estatus de herramientas de la mente, precisamente porque tienen capacidad para crear espacios que soportan sistemas de acción, no ya acciones aisladas. Por esta misma razón creemos también que nuestro enfoque supera la distinción que Salomon et al. hacen entre 
educación tienen las nuevas tecnologías, que no es otra que la posibilidad de incidir sobre los marcos de pensamiento, acción y representación del sujeto.

Vemos, pues, las nuevas tecnologías como un sistema de acción susceptible de ser utilizado para la realización de actividades educativas, o, si se prefiere, para la activación de procesos educativos; eso sí, siempre que sea pedagógicamente definido de antemano. Ésta es precisamente la función que debe cumplir el diseño de esos espacios que, por sus características técnicas, han dado en denominarse espacios virtuales de aprendizaje. Y difícilmente puede conseguirse esto si no reparamos previamente en los fundamentos, posibilidades y exigencias pedagógicas que deben cumplir esos nuevos escenarios o entornos. No estamos haciendo otra cosa que justificar con argumentos muy sencillos lo que parece obvio: la necesidad de explicar pedagógicamente los llamados entornos virtuales de aprendizaje, si realmente quieren serlo.

Hablando en términos estrictamente educativos, creemos que éste es el gran reto que hoy plantean las nuevas tecnologías y no, como suele ser habitual, los aspectos relativos a la cantidad de información susceptible de ser manejada y soportada, ni siquiera a la velocidad en la transmisión y uso de esa información y mucho menos los referidos a la superación de las limitaciones espaciotemporales a las que tradicionalmente estamos acostumbrados en educación, a no ser que esta característica se vea como condición imprescindible en la línea de nuestra propuesta. Éstas, y otras muchas funciones que sin duda cumplen muy eficazmente, decrecen en interés ante lo que consideramos que constituye el papel más relevante que hoy se asigna a la tecnología en educación y que no está tan orientado hacia el almacenaje, la recuperación, difusión y tratamiento de información cuanto a la promoción de la construcción de conocimiento y, más concretamente, a la creación de escenarios capaces de comportarse como catalizadores de procesos educativos que se correspondan con formas y tipos de aprendizaje que hoy se consideran socialmente valiosos. Quedarnos en aquellas funciones es reducir las nuevas tecnologias a sólo medios de instrucción y las formas de hacerlo son muchas, incluidas las que se reflejan en aquellos productos en los que la tecnología se comporta simplemente como tutor; preguntarse, en cambio, por la forma como crear y diseñar (Salomon, 2001) esos nuevos escenarios, en base a determinadas corrientes psicopedagógicas, de manera que se activen los mecanismos necesarios para favorecer, por ejemplo, la participación de individuos en sistemas de actividad concretos es, creemos, la cuestión de verdadero interés educativo.

\footnotetext{
"efectos con tecnologías" y "efectos de las tecnologías". Salomon, G.; Perkins, D. N. \& Globerson, T. (1991) Partners in cognition: Extending human intelligence with intelligent technologies, Educational Researcher, 20, 10-16.
} 


\section{UN MARCO DE INTELIGIBILIDAD PEDAGÓGICA DE LOS NUEVOS ENTORNOS DE APRENDIZAJE}

Entre los distintos tipos de alfabetización que los diversos campos disciplinares reivindican, hay algunos en los que coinciden todos ellos, puesto que son fruto de los cambios que les vienen afectando a todos más o menos por igual en las últimas décadas. Probablemente el más generalizado, sobre el que existe mayor grado de acuerdo, sea el que hace referencia a la alfabetización respecto de las nuevas tecnologías de la información y la comunicación. En este sentido en la literatura especializada comienza ya a ser clásica la diferenciación entre alfabetización tecnológica y alfabetización digital, no faltando quienes encuentran una especie de gradación progresiva entre ambos términos, señalando en ese caso otros tipos de alfabetización intermedios entre lo tecnológico y lo comunicacional, hablando, por ejemplo, de alfabetización informacional. Con independencia de la capacidad analítica para distinguir y matizar unos y otros tipos de alfabetización, la pedagogía, como campo disciplinar fuertemente afectado por estas tecnologías, debería reclamar la necesidad de contar con algún tipo de alfabetización exigible en aquellos casos cuya pretensión sea específicamente educativa. Ésta es nuestra intención.

La distinción entre alfabetización tecnológica y alfabetización digital se corresponde de alguna manera con los dos núcleos de funciones que al final del epígrafe anterior atribuíamos a las nuevas tecnologías, hablando en términos estrictamente educativos. Pero no nos podemos quedar aquí; es de gran interés, a su vez, distinguir dos líneas de reflexión en el análisis de la llamada alfabetización digital: de un lado, una línea que sigue de cerca los planteamientos de la teoría sociocognitiva sobre el aprendizaje y, de otro, una línea que utiliza la semiótica para estudiar el sentido, el significado y los comportamientos del sujeto ante los lenguajes propios de las nuevas tecnologías; ambas líneas de investigación caminan estrechamente unidas, pues los problemas que una plantea pueden encontrar solución en la otra y viceversa (Labbo, 2001). En este trabajo nos situamos conscientemente en la primera linea.

Decíamos más arriba que en estos momentos buena parte del llamado software educativo carece de soporte pedagógico y que, en ocasiones, las realizaciones y diseños telemáticos en uso se apoyan en interpretaciones teóricas insuficientes e incluso erróneas. Procedería ahora que echásemos un vistazo, en primer lugar, a aquellas interpretaciones y realizaciones telemáticas que resultan claramente insuficientes para explicar la acción y relación educativa. Es obvio que no lo vamos a hacer con la exhaustividad y extensión requerida ${ }^{4}$, pero sí aludiendo a un planteamiento muy usual en la actividad educativa cotidiana y, por tanto, suficientemente clarificador para nuestro objetivo.

4. Una buena síntesis de los mitos, leyendas y metáforas así como de las corrientes teóricas que subyacen a los usos e interpretaciones más habituales de estas tecnologías puede verse en Crook, Ch. (1998) Ordenadores y aprendizaje colaborativo. Madrid, Ministerio de Educación y Cultura-Ediciones Morata. 
Lo más habitual ha sido y es pensar en las nuevas tecnologías como instrumento al servicio del sujeto individualmente considerado, medios puestos a disposición del individuo en cuanto sujeto que lleva a cabo la actividad de aprendizaje en solitario. Sabemos que la imagen predominante, escolar, de la educación y del aprendizaje se corresponde con un proceso individual, que tiene lugar en la mente del sujeto y que se produce cuando no hacemos otra cosa; las tecnologías, las nuevas como las viejas, serían herramientas de facilitación y amplificación de ese proceso. Esta concepción, decimos, es predominante en los contextos institucionales de la educación, por más que en las dos últimas décadas esté siendo cuestionada por distintas corrientes y autores (Johnson, Johnson \& Holubec, 1999; Salomon, 2001; Wenger, 2001).

Precisamente una de las mayores potencialidades que presentan las nuevas tecnologías en la actualidad se refiere al desarrollo y fomento de todo tipo de tareas y actividades colaborativas, hasta el punto de que el entusiasmo por el trabajo con ellas se expresa, cada vez más, en términos de diseño de herramientas para la promoción de actividades colaborativas, participativas, cooperativas. Este extremo puede confirmarse fácilmente revisando la bibliografía de la última década en cualquier revista científica.

Pero conocemos también el efecto de refuerzo que en educación, como en otros campos disciplinares, se produce entre las imágenes teóricas predominantes sobre el aprendizaje y el uso que en correspondencia se hace de las herramientas y artefactos disponibles. Ésta es la manera de explicar que la práctica escolar lleve con mucha frecuencia a las nuevas tecnologías al mismo uso y destino que subyace en el planteamiento tradicional y predominante de la enseñanza, de forma que la inercia escolar termina reconvirtiendo el uso educativo de la tecnología en un sentido individual, solitario, mentalista y computacional. Con razón muchos profesionales echan en falta en estas tecnologías el ambiente personal e interpersonal, la calidad social del aprendizaje, ese plus de dinámica colectiva que va más allá de la gestión de la información procedente de los sistemas sensoriales y de los instrumentos de representación y manipulación de la información. Muchas de las corrientes teóricas que sustentan las realizaciones técnicas de las nuevas tecnologías no superan una interpretación de los procesos formativos como actividad independiente del sujeto, eso sí, gratamente experimentada por el usuario en base a la sensación de interactividad y autodominio que transmiten estas tecnologías.

Frente a estos planteamientos, que reducen los procesos educativos a mecanismos y dinámicas computacionales en base a símbolos y su interpretación, reglas y su aplicación, planes y su interpretación y aplicación, en definitiva, un juego de destrezas de pensamiento y herramientas cognitivas, las posibilidades que están abriendo las numerosas experiencias de trabajo y aprendizaje que se vienen haciendo con mediación de las nuevas tecnologías demandan una concepción teórica más amplia y apropiada, un marco de inteligibilidad que explique la dinámica que se crea en los entornos virtuales de aprendizaje teniendo como referencia los numerosos y. muy diversos componentes y mecanismos que conforman los procesos educativos en la actividad cotidiana. 
Y en cuanto enfoque global susceptible de proporcionar ese marco de inteligibilidad pedagógica, entendemos que el más apropiado es el denominado enfoque sociocultural, que acoge en su seno diversas corrientes con capacidad para contestar y formular las numerosas preguntas teóricas y empíricas que se suscitan en este campo. Este planteamiento centra su interés en los instrumentos de mediación y mecanismos de interacción social que configuran los sistemas de actividad de los sujetos, quedando entrelazados ambos aspectos, si bien la mayor parte de los estudios han enfatizado el segundo. Frente al individuo como unidad de análisis, propone los sistemas funcionales o sistemas de actividad como campo específico donde tienen lugar los procesos cognitivos y donde intervienen los individuos en interacción permanente con otros muchos y muy diversos elementos mediadores que estructuran la experiencia humana, físicos, simbólicos y sociales. Este enfoque se muestra más interesado en analizar la interacción del sujeto con el medio - personas, recursos materiales, simbólicos y sociales-, donde se desarrollan y conforman los procesos educativos, que en estudiar los procesos internos de pensamiento; más interesado en situar y analizar los marcos y las marcas culturales e históricas de esos procesos, la mediación social del proceso de configuración individual.

El énfasis que esta perspectiva coloca en el carácter inevitablemente mediador y social de los procesos formativos del sujeto conecta directamente con algunos de los interrogantes que a propósito de la utilización de las nuevas tecnologías en educación se formulan desde las posiciones más dispares, incluidas las correspondientes a los profesionales de la enseñanza. Más aún, muchas de las derivaciones de este planteamiento responden y tienen aplicación concreta a las posibilidades y exigencias que plantean las formas de hacer de las nuevas tecnologías. Pensemos, por ejemplo, en las implicaciones que para la configuración de los nuevos espacios de aprendizaje tiene el hecho de que la cognición, y por extensión toda la actividad formativa del sujeto, no se reduzca a procesos mentales íntimos, sino que se resuelva en términos de sistemas de actividad conformados por la acción de las personas en entornos y situaciones específicas, donde se encuentran desparramados los diversos artefactos y herramientas, físicas, simbólicas y sociales; o en las consecuencias que para el diseño de esos nuevos espacios ha de tener el carácter situado y distribuido, implícito en la afirmación anterior, de toda nuestra cognición y pensamiento, ya que conocer es un actividad y no una propiedad y no se da fuera de ese complejo interaccional que incluye inevitablemente elementos externos al sujeto o, por último, la naturaleza profundamente social de todos los procesos cognitivos, no sólo en el sentido de que los elementos mediadores son históricos sino por el hecho de que se actualizan en las relaciones interpersonales de los sujetos, de manera que la adquisición de conocimiento se produce inevitablemente a través de la participación de los sujetos en determinados tipos de interacción social ${ }^{5}$.

5. Una de las ideas fuerza de este enfoque viene dada por el concepto vygotskiano de zona de desarrollo próximo, originariamente interpretado en términos de participación y colaboración, bastante 
Estas implicaciones, entre otras, nos están invitando a una reconsideración conjunta de la naturaleza del conocimiento, las formas como se producen los procesos educativos y las concepciones y usos de las nuevas tecnologías en educación. Se nos abre así una interesante línea de reflexión pedagógica sobre las formas como actúan las nuevas tecnologías y, en última instancia, sobre las formas como tienen lugar los procesos formativos en los entornos virtuales y las consecuencias que ello tiene para su diseño y configuración en términos pedagógicos.

A partir de ahora nos centraremos precisamente en esto, comenzando por presentar algunas concreciones teóricas del planteamiento histórico-cultural de la cognición y el aprendizaje cuyas implicaciones pedagógicas para el diseño y la configuración de los entornos virtuales analizamos posteriormente ${ }^{6}$

\section{LA FORMACIÓN EN LOS ENTORNOS VIRTUALES DE APRENDIZAJE: CONSIDERACIONES TEÓRICAS}

Un entorno virtual de aprendizaje puede ser visto como un espacio de formación en el que se utilizan canales de comunicación mediatizados por la tecnología, fundamentalmente basada en la web, que tiene como fin la consecución de un proyecto educativo contextualizado. En ocasiones este proyecto surge a partir de un tema de interés común para un grupo o de un problema que admite múltiples soluciones que pueden ser aportadas por cada uno de los participantes en alguna de las zonas de actividad definidas en el sistema.

Sin entrar en los componentes que tienen que ver con las características informáticas o con los recursos telemáticos contenidos en la noción de virtualidad y centrándonos en aquellos otros más genuinamente pedagógicos, en esta sencilla aproximación inicial al concepto de entorno virtual se observan varios elementos básicos que lo caracterizan: la definición de un sistema de actividad colaborativa, la presencia de zonas de desarrollo próximo identificadas por los diferentes niveles de competencia de cada uno de los sujetos participantes, el concepto de interacción y la idea de contextualización. Todos estos elementos están íntimamente relacionados entre sí y configuran el marco teórico que entendemos debe considerarse de cara al diseño de la formación en un entorno virtual de aprendizaje y que sintetizamos en lo que sigue en torno a los conceptos de colaboración, actividad y comunidad.

más allá de la mera presentación y tratamiento computacional de la información, ya sea personal o artificialmente. La metáfora bruneriana del andamiaje insiste en la misma idea de participación, encuentro, actividad conjunta, coordinación y cooperación.

6. Los epígrafes que siguen provienen, además de una amplia revisión de la literatura sobre el tema, de nuestra experiencia y reflexión sobre el desarrollo y aplicación de dos entornos virtuales de aprendizaje que hemos denominado Tutor-pea y Tutor-te. El primero dirigido a la formación permanente del profesorado de educación de adultos; el segundo, a personas mayores. 


\subsection{El concepto de colaboración en los entornos virtuales de aprendizaje}

El aprendizaje colaborativo impregna la mayor parte de los planteamientos teóricos que han analizado la aplicación de las TIC a la educación, en particular, aquellos enfoques que enfatizan el papel social del aprendizaje. En parte, esto es así porque la cooperación y, más aún, la colaboración es considerada como uno de los rasgos con mayor poder integrador y valioso del planteamiento formativo en cualquier entorno de aprendizaje ${ }^{7}$.

La colaboración alude etimológicamente a la idea de "trabajar juntos o con otros". En esta noción está implícita la idea de que varias personas, de diferentes niveles de competencia, realizan una o más actividades con objeto de obtener un beneficio mutuo y tratar de alcanzar un objetivo común. En el terreno educativo la colaboración se entiende como una estructura de interacción que conduce al aprendizaje y a la construcción de conocimiento mediante un proceso sinérgico de apoyo e interacción grupal, cuya eficacia descansa necesariamente en el compromiso individual, la responsabilidad mutua y la interdependencia positiva entre los miembros del grupo (Johnson y Johnson, 1994).

Pero además de con los otros, el concepto de colaboración alude al hecho de que se aprende de los otros. Visto desde la teoría socioconstructivista, el conocimiento no es un objeto que se pasa de un sujeto a otro sino que es algo que se construye por medio de operaciones y habilidades cognoscitivas que se inducen en la interacción social. En la práctica educativa esto implica disponer un sistema de relaciones entre los miembros del grupo en el que debe darse un intercambio de roles y tareas que posibiliten la co-construcción y la apropiación de conocimientos, permitiendo a los sujetos la construcción e interpretación conjunta de la realidad, dotando de significado a los contenidos a aprender.

La aparición y notable desarrollo del enfoque CSCL (Computer-Support Collaborative Learning) desde mediados de los años 90 (Koschmann, 1996; Dillenbourg, 1999; Gerry, 2002) ha abierto nuevas perspectivas sobre el análisis de la colaboración en los entornos e-learning, conjugando los aspectos sociales, psicológicos, organizacionales y sus efectos sobre el aprendizaje. Más particularmente, la perspectiva CSCL se ha interesado principalmente en determinar la forma como el aprendizaje colaborativo asistido por las tecnologías puede mejorar la interacción entre pares y el trabajo en grupo o en equipos; cómo la colaboración, así como la tecnología, facilitan el conocimiento compartido y distribuido, además del desarrollo de habilidades y destrezas entre los miembros de la comunidad (Waldegg Casanova, 2002). En síntesis, la orientación introducida por este paradigma en la educación enfatiza la idea de que la dinámica grupal colaborativa está

7. Entendemos aquí el concepto de aprendizaje cooperativo subsumido en el de colaboración. Ambos conceptos están, en cualquier caso, unidos, a pesar de que exista una cierta controversia a la hora de distinguirlos o no. 
estrechamente relacionada tanto con una adecuada interactividad como con una positiva interacción social dentro del sistema.

La interactividad rompe la linealidad narrativa propia de los sistemas textuales, proporcionando al usuario la posibilidad de establecer sus propios itinerarios de navegación a través de la estructura hipertextual que le presenta la interfaz. El objetivo de cualquier entorno interactivo es buscar la implicación activa del sujeto en las tareas que son propuestas en el sistema. Esta actividad recíproca que se establece entre los contenidos de aprendizaje o entre el ordenador y el sujeto exige el diseño adecuado de herramientas técnicas que permitan el intercambio fluido de información, experiencias, conocimientos... La idea es que, cuanto más interactivos sean los materiales de enseñanza, más enriquecedora y de mayor calidad será la colaboración y, en consecuencia, mejor será la relación entre los usuarios de cara a favorecer el proceso de construcción social del conocimiento y el aprendizaje. En la medida que el sistema sea más "cálido", atractivo para el sujeto, mayor y mejor será también la relación entre éste y el ordenador.

Cuando se establece el diálogo entre el usuario y la máquina, se activan mecanismos de tipo perceptivo visual, cognitivo, actitudinal y motivacional, además de la propia interacción física con el artefacto, que provocan, en conjunto, una reacción más o menos positiva del sujeto hacia el sistema con un notable efecto en su toma de decisión sobre la continuidad o no en el mismo. Por eso la interactividad del sistema viene dada por la calidad de la interfaz y de la aplicación informática utilizada en cada caso, así como por la intencionalidad del diseñador en buscar o no dicha interactividad. Con todo, entendemos que la búsqueda de calidad técnica de la interfaz y, en consecuencia, de la mejora de la relación ordenador-individuo no es en absoluto incompatible con la idea expresada por Bates $(1995,227)$ de que la tecnología pase lo más desapercibida posible, sea "invisible".

Si la interactividad afecta la relación ordenador-usuario y, en última instancia, la del contenido-alumno, la interacción busca generar una comunidad social virtual. Esto implica favorecer un sistema de apoyo grupal en el que se fomente una relación amigable entre los miembros de los grupos, generando un ambiente de confianza. Conviene señalar que éste es uno de los aspectos de mayor dificultad en la práctica del trabajo colaborativo en ambientes virtuales. Para ello es necesario que la estructura técnica diseñada para la comunicación en el entorno virtual cuente con algunas herramientas - tipo grupos de discusión, chat, espacios lúdicos o de ocio...- que tengan como uno de sus objetivos principales precisamente favorecer este tipo de componente de refuerzo interpersonal y, en definitiva, incrementar la calidad y la cantidad de participación de los intervinientes ${ }^{8}$.

8. La participación en los entornos virtuales suele estimarse por el número de veces que el sujeto interviene, rango de actividades en que participa, relevancia de las actividades en que se involucra, grado de satisfacción del sujeto y del grupo, etc. 
Desde nuestra propia experiencia hemos podido comprobar que la interacción en estos espacios precisa un nuevo sistema de comunicación que mitigue el efecto de la pérdida de comunicación no verbal que se da en el contexto presencial. La ausencia de lenguaje y/o comunicación corporal propio de la presencialidad precisa otro tipo de elementos sustitutivos que hacen necesaria una mayor explicitación de las instrucciones y las tareas. La inclusión de imágenes personalizadas, fotografías y datos personales de los usuarios resulta pertinente y necesario, aunque tal vez no suficiente, por lo que se precisan adecuados materiales instructivos, recursos didácticos personalizados que faciliten el proceso de participación, de interacción y de mediación.

Además de adecuados materiales, la interacción en un entorno virtual se consigue mediante el desarrollo de un proceso de comunicación basado principalmente en el diálogo y la argumentación. Ahora bien, dado que el aprendizaje colaborativo en estos espacios se promueve generalmente mediante sistemas de trabajo poco estructurados, en la práctica es necesario articular una secuencia instructiva que permita la adquisición de conocimiento mediante esa discusión y el intercambio de perspectivas entre los miembros del grupo. Resulta, pues, de interés iniciar siempre el proceso de aprendizaje a través de la discusión guiada, donde "se lleva" al sujeto a participar activamente en los grupos de debate o los foros de discusión de manera directa o vicariamente, observando y reflexionando sobre las intervenciones de los demás participantes. En suma, el intercambio de puntos de vista, la negociación de perspectivas, la reconstrucción de significados es la base del aprendizaje en el entorno virtual, por encima de la transferencia de información o el almacenamiento de documentos. Este intercambio de información y de reflexión de unos y otros en la interacción social conduce a la modificación de los esquemas de interpretación, produciéndose el necesario conflicto cognitivo que posibilita el aprendizaje y el nuevo conocimiento.

\subsection{El concepto de actividad en los entornos virtuales de aprendizaje}

Acabamos de manifestar que una de las claves a la hora de diseñar un adecuado entorno virtual de aprendizaje colaborativo pasa por conseguir una buena interacción entre los integrantes del sistema. Pero, ¿qué significa en términos pedagógicos interactuar en este tipo de entornos de aprendizaje? Desde la teoría vygostkiana, la interacción suele explicarse a partir del concepto de zona de desarrollo próximo y, por extensión, puede aplicarse a la relación que se establece entre las diferentes zonas de los distintos participantes, desde el momento en que un sujeto cualquiera hace uso de los conocimientos, información, datos, reflexiones... acumulados en el sistema por otros usuarios.

$\mathrm{Al}$ analizar este tema, algunos autores matizan que, para conseguir una adecuada interacción, resulta necesario determinar hasta qué punto deben "coincidir" las zonas de desarrollo próximo, de modo que el trabajo del grupo sea eficaz; es 
decir, que el grupo sea lo suficientemente homogéneo como para que sus ZPD tengan puntos de coincidencia y se produzca así el intercambio de conocimientos, pero lo suficientemente heterogéneo también como para que unos sujetos puedan aprender de otros. Es éste un aspecto básico que nos remite nuevamente a la idea de la confianza mutua entre los participantes. R. Lewis (2001) señala al respecto que la confianza en el aprendizaje reviste la misma importancia que en el puesto de trabajo y que en general suele existir una cierta desconfianza de los estudiantes en los conocimientos o aptitudes de sus compañeros. También desde nuestra experiencia hemos tenido ocasión de comprobarlo. La fijación de ciertos hábitos escolares que consideran como único conocimiento válido el que proviene del profesor, a veces incluso por encima del de otras fuentes de información, sigue siendo muy fuerte entre muchos estudiantes y dificulta una mayor disposición del alumno a aceptar "la ayuda", esto es, los conocimientos de otros iguales a él. Dicho de otro modo, impide "entrar" en la zona de desarrollo de los demás, al tiempo que "abrir" su propia zona para que los demás puedan beneficiarse de esta ayuda; en suma, facilitar el proceso de apropiación.

Para la teoría pedagógica ésta es una cuestión clave. Se expresa en la preocupación sobre el modo de generar zonas de desarrollo próximo colectivas o, en expresión de Newman, Griffin y Cole (1991) zonas de construcción del conocimiento, denominadas también por otros autores zonas comunes de intersubjetividad. Entendemos que en los contextos e-learning el modo de generar este tipo de entornos de aprendizaje colectivo o cómo compartir esta intersubjetividad es el gran asunto. El uso de estrategias y metodologías basadas en dilemas, conflictos cognitivos, actividades dialógicas y metodología conversacional puede ser un buen recurso instructivo, conscientes de que la utilización de este tipo de metodologías en favor de la intersubjetividad en el sistema implica generar un doble sistema de actividad mediatizada por el ordenador, de interacción y de interactividad.

La noción de actividad es ya antigua, remite a la revitalizada ahora tradición sociocultural soviética, a partir de los trabajos de autores como Luria, Vygostky, Leontiev y seguidores. Las ideas básicas son suficientemente conocidas. En síntesis, la actividad humana se entiende como un conjunto de procesos socialmente mediatizado por determinados símbolos, signos, artefactos o instrumentos que son elaborados histórica y culturalmente. A través de esa actividad cultural mediada y de la propia interacción con los demás, el sujeto construye y reelabora su conciencia, así como su visión del mundo y, en consecuencia, su acceso, que resulta ser indirecto y mediado, a la realidad.

A esta interpretación de la actividad basada en la tradición socioconstructivista clásica se han sumado, en el contexto del paradigma CSCL, los trabajos de autores como Engeström (1987), Engeström y Middleton (1996), McLellan (1995), Dillenbourg y Self (1992), así como otras tradiciones de contenido más sociológico con base en la etnografía, la antropología cultural y cognitiva, el interaccionismo y sobre todo la etnometodología, que enfatizan la importancia de la influencia de los contextos sobre cualquier actividad social de los individuos, dando lugar, todo ello, a 
las perspectivas sobre la cognición situada (Suchman, 1993; Brown et al., 1989; Lave y Wenger, 1991; McLellan,1995; Clancey, 1999) y de la cognición distribuida (Suchman, 1987; Lave, 1991; Norman, 1993; Hutchins, 1995; Pea, 1993; Salomon, 2001)9.

El aumento de perspectiva que sobre el concepto de actividad introducen las teorías del aprendizaje situado y de la cognición distribuida conduce a asumir los escenarios profesionales y/o educativos como sistemas o espacios de actividad en los que la interacción es vista como un proceso integrado y distribuido entre todos los elementos presentes en la situación: el espacio y el tiempo, los artefactos del entorno, así como la percepción y la cognición conjunta que se establece en la comunicación o en la interacción de los sujetos participantes. En síntesis, desde el concepto de aprendizaje situado, propuesto por J. S. Brown et al. (1989), a partir de la teoría sociocultural de Vygostky, se alude a una forma de actividad que asume la importancia del contexto, de la cultura en la que es realizada, sin la cual no puede ser abstraída dicha conducta o actividad. Lo sustantivo aquí es que la actividad, y en realidad cualquier práctica social, está situada en un contexto específico y además el conocimiento que surge o que está presente en ese contexto se encuentra socialmente distribuido. Desde la idea de distribución de la cognición o de la inteligencia se quiere significar que los recursos que dan forma a la actividad y la hacen posible están distribuidos en configuraciones entre las personas, los entornos y las situaciones; dicho de otra forma, la inteligencia se concibe no como un atributo individual, como una cosa que se posee o una propiedad del ser, sino como algo que se ejerce, que se manifiesta en la actividad, en la acción. En este sentido, las nuevas tecnologías se conceptúan como reorganizadoras del funcionamiento mental y no meramente como amplificadoras de la cognición, proporcionando a las personas recursos para orientar la actividad e incrementarla (Pea, 1993).

El modo de orientar e incrementar la actividad situada y distribuida en el entorno virtual, a la hora de implementar un modelo formativo de interacción, que permita al grupo explorar, analizar, reflexionar, proponer, actuar y sobre todo compartir, pasa por dotar de significado a la relación enseñanza-aprendizaje, posibilitando el principio básico de transferencia del conocimiento a situaciones de la vida real/profesional de los participantes. En suma, nos estamos refiriendo a que una característica básica del sistema debe ser la contextualización. Naturalmente el planteamiento didáctico en base a supuestos tomados de la práctica real implica una simulación o representación figurada de la realidad, pero es precisamente esta

9. El aprendizaje y la coginición situada es el estudio cle la coginición dentro de su contexto natural. Esta perspectiva enfatiza que la mente de los individuos normalmente opera dentro de ambientes que estructuran, dirigen y apoyan los procesos cognitivos. Este ambiente o contexto puede ser definido como físico o basado en tareas, incluyendo artefactos y representaciones externas de la información, ambiental o ecológico, social o interaccional, como el contexto educativo instruccional o los ambientes clínicos. Para más detalle véase LOzARES, C. (2000) La actividad situada y/o el conocimiento socialmente distribuido, Papers, Revista de Sociología, 62, 97-131. SAlomon, G. (comp.) (2001) Cogniciones distribuidas. Consideraciones psicológicas y educativas. Buenos Aires, Amorrortu. 
contextualización la que permite la capacidad de transferencia de los resultados de la actividad conjunta desarrollada, así como la resolución de nuevos problemas. Con todo, en determinados contextos formativos, como los configurados por los entornos virtuales, promover la sensación de una enseñanza contextualizada no resulta fácil. Una opción metodológica eficaz es partir de una situación problematizada que, a través de la discusión y la reflexión conjunta, va derivando hacia actividades o tareas que son propuestas por cualquier participante en el sistema.

La consideración de la interacción social colaborativa y la actividad como bases del aprendizaje, apoyadas en la idea de contextualización y las nociones de aprendizaje situado y de cognición socialmente distribuida, remiten a un último elemento que queremos considerar en este apartado y que, en gran medida, integra a los anteriores, confiriendo mayor coherencia teórica al diseño pedagógico de los espacios de formación virtual. Nos referimos al concepto de comunidades de aprendizaje o comunidades de práctica.

\subsection{Los entornos virtuales de aprendizaje como comunidades de aprendizaje o de práctica}

La argumentación anterior nos lleva a un punto en el que se resalta que la actividad formativa en los entornos virtuales de aprendizaje pasa necesariamente por generar situaciones de práctica, real o simulada, desarrolladas en el marco de un grupo de trabajo o también de una comunidad de aprendizaje ${ }^{10}$. La línea de trabajo y de reflexión conducida especialmente a partir de los trabajos de Jeane Lave y Etienne Wenger en torno a la noción de comunidades de práctica proporciona un ejemplo particularmente sugestivo del trabajo de colaboración distribuido en espacios socioculturalmente definidos, con un especial interés en su derivación hacia los entornos virtuales de aprendizaje.

Para Lave y Wenger (1991) en las comunidades de práctica el aprendizaje se construye cuando se comparten actividades significativas, siendo este tipo de comunidad una condición intrínseca para que ocurra el conocimiento. Desde esta perspectiva se asume que el aprendizaje es inseparable de la práctica, de forma que el que aprende no sólo se acerca al conocimiento sino a todo un conjunto de prácticas sociales y a los valores que a ellas se asocian (Lave, 2001) ${ }^{11}$. Dicho de otro

10. El concepto de comunidad de aprendizaje ha sido caracterizado por algunos autores como espacios compartidos en los que se genera una atmósfera de responsabilidad individual, junto con el sentimiento de que algo está siendo compartido, de manera que los participantes se insertan en un sistema social en el que se crea una comunidad de discurso y de crítica constructiva; el estudiante asume su rol como principal responsable de su propio aprendizaje y el profesor el de mediador, introductor y facilitador del aprendizaje (BRown, Collins \& Duguid, 1989).

11. Lave señala que para constituir comunidades de práctica es necesario, en primer lugar, que los participantes tengan un acceso amplio a las diferentes partes de la actividad y, eventualmente, participen de forma plena en el núcleo de las tareas. En segundo lugar, que se dé una interacción horizontal 
modo, las interacciones y la colaboración entre participantes en entornos virtuales de aprendizaje se entienden, desde este concepto, como un proceso de participación en comunidades de conocimiento. Este conocimiento surge a partir de un proceso informal que se configura en la práctica diaria de una comunidad, mediante el acto de compartir determinadas historias de aprendizaje (Wenger, 2001, 115).

La condición de "informalidad", inherente a las comunidades de práctica, choca con el discurrir habitual de los contextos educativos presenciales, más estructural e intencionalmente formales. De ahí que la aplicabilidad de este concepto a la educación puede verse facilitada precisamente por el uso de herramientas y entornos tecnológicos en los cuales, por definición, las limitaciones espacio-temporales son menores y permiten generar sistemas abiertos de trabajo, similares a los del mundo laboral, en los que se promueven planes de acción, asumidos por el grupo, o generan redes informales en las que fluyan las ideas, los conocimientos, las opiniones, las experiencias vitales y las vivencias de participación de cada sujeto. En definitiva, las comunidades de aprendizaje electrónicas pueden proporcionar oportunidades para mediar en algunos procesos de aprendizaje que sí están presentes en los escenarios sociales informales y que resultan menos accesibles en los contextos presenciales clásicos, donde quizá sea más difícil romper determinados hábitos y estructuras de interacción. Precisamente el concepto de práctica destaca el carácter social y negociado de lo explícito y de lo tácito que hay en la vida de cada sujeto individual, que en determinados contextos, particularmente en los laborales, se apropia de todo el repertorio compartido y producido de la comunidad (rutinas, palabras, instrumentos, maneras de hacer, relatos, símbolos, acciones o conceptos).

Estas ideas pueden y deben ser tenidas en cuenta en el diseño de la formación en lós entornos virtuales de aprendizaje. Reiteramos que un recurso básico para la creación de una comunidad virtual de aprendizaje o, si se quiere, de práctica, es el diálogo. El diálogo entre dos o más sujetos, a través de un proceso de construcción guiada, de intercambio, distribución y compartición de saberes o conocimientos, se convierte en el paso previo para la reflexión individual y para el pensamiento crítico, permitiendo una mayor profundización en los temas de estudio o de trabajo. El razonamiento y el cuestionamiento, por encima de la actividad memorística o sin significación para el sujeto, se convierten así en los elementos básicos de una metodología fundamentada en la discusión.

Este enfoque conversacional, aprendizaje conversacional (Kolb, 1998) o discusión argumentativa (Van Joolingen, 2000), resulta ciertamente interesante y conecta fácilmente con otras líneas de trabajo clásicas, especialmente en el ámbito

\footnotetext{
entre ellos, especialmente en situaciones problemáticas a las que, además, se les busque una solución. En tercer lugar, dotar de transparencia a las estructuras y tecnologías de la comunidad de práctica, es decir, que el trabajo esté disponible para los participantes. Puede verse a este respecto Lave, J. (1991) La cognición en la práctica. Barcelona, Paidós. Ídem (2001) La práctica del aprendizaje, en Chaikun, S. \& Lave, J. Estudiar las prácticas. Perspectivas sobre actividad y contexto. Buenos Aires, Amorrortu.
} 
de la educación de adultos, como son las inspiradas en la teoría de Paolo Freire en el marco de las denominadas comunidades dialógicas. En este marco, la conversación, la discusión y la reflexión dialogada, implementadas a través de un proceso de construcción guiada, constituyen un elemento nuclear para promover el aprendizaje. Insistimos en que en los contextos e-learning son especialmente trasladables este tipo de ideas, dado que la inmediatez en la búsqueda y localización de información así como la facilidad de intercambio de opiniones o reflexiones mediante la aplicación de distintas herramientas permiten no demorar la resolución de determinadas dudas y facilitar los procesos de interacción entre agentes muy diversos, haciendo más efectivo el aprendizaje y generándose, en suma, un importante potencial cognitivo que puede ser convenientemente aprovechado en un entorno virtual de aprendizaje.

\section{CONFIGURACIÓN DE LOS ENTORNOS VIRTUALES DE APRENDIZAJE: INTERPRETACIÓN PEDAGÓGICA}

Desde una u otra corriente teórica, ya partamos de la noción más amplia de comunidad de práctica, enfaticemos el concepto de colaboración o hagamos resaltar la idea de actividad situada y distribuida, llegamos a un punto de confluencia que es cómo configurar los entornos virtuales de aprendizaje de manera que se produzcan esos tipos y formas de actividad y cognición cuyo núcleo conceptual gira en torno al concepto de interacción. $O$, si se prefiere, y hablando de entornos configurados tecnológicamente, el aspecto central es una cuestión de diseño de espacios, virtuales por supuesto.

A este respecto, antes de seguir adelante con nuestra reflexión referida específicamente a la estructura y funcionamiento de uno de los entornos virtuales que hemos desarrollado ${ }^{12}$, vamos a hacer un comentario a propósito de una actividad cotidiana, muy acorde, por lo demás, con la metodología característica de los estudios socioculturales, sea cual fuere la posición teórica concreta. Pensemos en una actividad que todos hemos hecho y hacemos con frecuencia. Todos hemos entrado numerosísimas veces en una de esas grandes superficies llamadas genéricamente supermercados, en uno de esos grandes espacios comerciales donde la primera sensación es de desorden, barullo, amontonamiento y descolocación generalizada de las personas y de los productos. Todos hemos entrado, nos hemos movido por su interior durante largos ratos, con frecuencia más tiempo del previsto, hemos comprado muchas cosas, con frecuencia más cosas de las que pensábamos... y todo ello sin detenernos en ningún momento a pensar en el porqué de nuestro comportamiento. Si así fuere, caeríamos en la cuenta de que no es posible comprar tantas

12. «Tutorpea. Formación permanente de profesorado de educación de adultos". Acción financiada por la Comisión de las Comunidades Europeas. http://www3.usal.es/tutorpea/, http://laboreduca.usal.es/sistema_tutor/principi.htm. 
cosas en tal período de tiempo, si los espacios y los productos no estuviesen bien organizados, distribuidos y situados.

Lo que todos realizamos tantas veces de una manera que aparentemente podíamos calificar de natural no lo es tanto. Alguien, los especialistas en marketing, diseño de interiores, etc. - -siempre hay detrás de nuestros comportamientos algún especialista, o antes o después-, han pensado, en este caso mucho antes, por nosotros $y$ han organizado y distribuido espacios y colocado productos en función de unos criterios, de manera que han convertido la acción cotidiana de comprar en un sistema de actividad e incluso, dirán algunos, en una práctica de comunidad. En todo caso, parece obvio que la compra no es ningún acto reflexivo o espontáneo, según se mire, y que, manipulando el espacio en el que se mueven los sujetos, se manipula a su vez la otra coordenada que resulta imprescindible para el sujeto, o sea el tiempo, y que a través de esas coordenadas se termina manipulando en definitiva la actuación y el comportamiento de los sujetos. Este comentario a propósito de los espacios comerciales bien puede extenderse respecto de otros espacios donde habitualmente se mueve el sujeto.

Pues bien, ¿es posible trasladar algunos términos de este planteamiento a los denominados espacios virtuales de formación?, ¿no será esta vía precisamente uno de los caminos por donde conseguir la marca específicamente educativa de los llamados espacios virtuales?, ¿qué significa "diseño" en términos pedagógicos?, ¿y aplicado a los espacios virtuales?, ¿tiene ello las mismas implicaciones y supuestos pedagógicos que si estuviéramos hablando de diseño de espacios e instrumentos en contextos presenciales? Estas y otras preguntas análogas vienen preocupándonos hace tiempo y han cobrado protagonismo a partir de las experiencias ya citadas que recientemente hemos tenido con entornos virtuales de aprendizaje. Hemos de reconocer, sin embargo, que no tenemos tantas respuestas como preguntas, pero unas y otras son importantes y hasta imprescindibles, en cuanto que proporcionan tareas a la investigación.

Desde los primeros momentos de nuestra investigación fuimos conscientes de que nos estábamos moviendo en el ámbito del diseño, aplicación y desarrollo de instrumentos y estrategias, técnicas y pedagógicas. Pero poco a poco caímos en la cuenta de que esta tarea, en el marco de las nuevas tecnologías, iba más allá del diseño de instrumentos y bien podía traducirse en términos de disposición y distribución de espacios y recursos; dicho de otra manera, diseñar entornos virtuales de aprendizaje es, entre otras cosas, tomar decisiones en relación con la distribución y disposición de espacios, tipos y modos de espacios y de movimientos y acciones en los espacios, interpretado todo ello en términos de realidad virtual ${ }^{13}$.

13. ¿Quiere ello decir que es posible y suficiente, cuando nos movemos en terrenos virtuales, con trasladar el esquema mental de los tipos y modos de acción de la actividad presencial? Nunca lo vimos así, al contrario, entendemos que las nuevas tecnologías tienen que proporcionar un plus de valor añadido, ya sea cuantitativa o cualitativamente, a las potencialidades educativas de la formación presencial, lo que probablemente exigirá también un plus de requerimientos pedagógicos. 
Y para ello necesitábamos un marco de inteligibilidad que hemos encontrado de forma general en el enfoque sociocultural y, más concretamente, en las corrientes teóricas que acabamos de recuperar en epígrafes anteriores. Con mucha razón algunos autores (Crook, 1998) entienden que ese enfoque ofrece las mayores posibilidades para "inferir las mejores formas de desplegar la nueva tecnología", hasta el punto de que los espacios virtuales de aprendizaje, su diseño, configuración y distribución, pueden ser vistos como la materialización de las llamadas zonas de construcción del conocimiento. En términos más generales, podríamos decir que los entornos virtuales de aprendizaje, su diseño y configuración, pueden ser concebidos e interpretados pedagógicamente en base a muchos de los conceptos, mecanismos y funcionamiento resaltados por las distintas corrientes que han desarrollado el pensamiento originario de Vigotsky. Esto es precisamente lo que queremos reflejar a continuación; lo vamos a presentar de una manera analítica y progresiva, haciendo corresponder nuestra reflexión con algunos aspectos, mecanismos y funcionamientos concretos de los entornos virtuales en los que hemos trabajado. Comenzaremos por lo que nos parece más obvio.

Cabe pensar que si la cognición, la mente, el conocimiento y el aprendizaje están, se presentan y funcionan como algo distribuido, la manera concreta como lo están depende, al menos, del entorno o marco en que se encuentran, el tipo de actividad que se desarrolla, los instrumentos mediadores y los objetivos que se persiguen (Lave, 1991; Cole y Engeström, 2001). Ahora bien, en el contexto de las nuevas tecnologías, dado que ellas son susceptibles de generar entornos o espacios específicos, la mayor dependencia de la distribución de las cogniciones y del aprendizaje resultante estará en función de los objetivos, tipos de actividad y medios utilizados. Dicho de otra manera, las formas como en los entornos virtuales se puede materializar esa distribución es a través de los espacios y, sobre todo, recursos y actividades que permitan poner en marcha y hacer intervenir, formas concretas de actividad que se buscan y variedad de recursos que se hace intervenir.

$\mathrm{Si}$, además, la pretensión última del planteamiento es de tipo colaborativo, como en el caso del entorno que hemos denominado Tutorpea, puede concluirse, en principio, que cuanto mejor sea la estructura de interacción diseñada, en el sentido de una notable y bien pensada distribución de espacios, actividades y recursos, mayores serán las oportunidades para potenciar la participación, el encuentro y la vertiente colaborativa de la interacción social ${ }^{14}$.

14. En el fondo de este principio subyace la idea de habitabilidad de los entornos virtuales, su capacidad e invitación a la acción y relación. "To a large extent, the future success of virtual communities depends on how well the tools for social interaction are designed. If they are poorly designed, the online world may feel like a vast concrete corporate plaza, with a few sterile benches: a place people hurry through on their way to work or home. If the tools are well designed, the on-line world will not only be inhabited, but will be able to support a wide range of interactions and relationships, from close collaboration to casual people watching". DONATH, J. S. (1997) Inbabiting the Virtual City: the design of social environments for electronic communities. Massachusetts, MIT, p. 11. Este planteamiento se ha corroborado en 
Apoyándonos en este principio, podríamos decir que pedagógicamente procede que los entornos virtuales ofrezcan técnicamente libertad y diversidad de movimientos, actividades y recursos ${ }^{15}$, en cuanto que de esta manera proporcionan mayores puntos posibles de encuentro a través de los cuales se puede catalizar la actividad colectiva, social, y materializarse la colaboración. Esta libertad y diversidad de movimientos, muy aconsejable en entornos virtuales, se asemeja más a las formas de desenvolvimiento y actuación en contextos informales que a las propias de los contextos escolares. Esta observación es importante, en cuanto que entendemos más fructífero para la investigación técnico-pedagógica este traslado a los espacios virtuales de las formas y recursos de acción que se producen en los contextos cotidianos de actividad, por ejemplo, saltos no precisamente lógicos que terminan encadenando la acción, mejor, decimos, que intentar trasladar las formas de actuar propias de los espacios escolares.

Dando un paso adelante en nuestra reflexión, queremos detenernos ahora en otro de los principios básicos del enfoque sociocultural, ignorado o al menos minusvalorado tradicionalmente y recuperado de una u otra manera por las distintas corrientes que actualmente desarrollan este enfoque. Lo hacemos por entender que su aplicación constituye uno de los soportes fundamentales de los entornos virtuales de aprendizaje, aunque habitualmente, como decimos, no suele tenerse en cuenta al presentar la dimensión social del aprendizaje, de la cognición o de la actividad formativa en general. Nos estamos refiriendo a que lo más usual es hacer reposar ese basamento social en la relación e interacción entre personas.

Los entornos virtuales, en cambio, y concretamente el entorno virtual que ofrece Tutorpea, se apoyan originariamente en una segunda interpretación no menos vygotskiana, una interpretación de la trama social no tan interpersonal cuanto comunitaria, societaria, en el sentido de hacer reposar su estructura técnicosocial en los recursos y formas como los grupos y las comunidades organizan ambientes donde desarrollan actividades para conseguir algo. Dicho con otras palabras, en los entornos generados con la mediación de las nuevas tecnologías se crea técnicamente un ambiente dispuesto de forma tal que se provoquen interacciones, apoyándonos para ello en unos componentes previos a la relación interpersonal, componentes que van a mediar esa relación y que vienen condicionados por las

el funcionamiento del Tutorpea de otras maneras, entre las que no queremos pasar por alto la siguiente: una vez diagnosticado el tema o problema que iba a ser tratado, el nivel de participación así como de reflexión conjunta aumentaba en la medida que se incrementaba y diversificaba el número y tipo de tareas a realizar. De alguna manera estaríamos ante la misma idea: la colaboración necesita espacios de participación que provoquen encuentros, aunque sean fortuitos en una primera fase. Algo pareciclo ocurre cuando hablamos de colaboración en contextos presenciales.

15. El sistema Tutorpea permite entrar, salir y moverse por distintos lugares: hacer preguntas, más o menos urgentes, de uno u otro tipo; proponer temas, problemas, situaciones; configurar progresivamente el tema propuesto aportando matices a las intervenciones de los demás; aportar formas de tratamiento de problemas; discutir informalmente con otros participantes; intervenir en grupos de discusión más formales.. 
posibilidades técnicas y las pretensiones sociales propias de la configuración del espacio de que se trate. Ello significa que, en los procesos formativos que tienen lugar mediante entornos virtuales, la metáfora bruneriana del andamiaje no debe utilizarse solamente para referirnos a la construcción de la relación interpersonal, pues también es útil en esa otra interpretación más societaria que acabamos de recuperar; diríamos que ésta en algún sentido es previa a aquélla. Lo que se andamia, en realidad, son muchas cosas, empezando por la disposición del entorno de forma tal que provoque tramas de participación y coordinación de actividades; en base a símbolos y artilugios configuradores y delimitadores de espacios y actividades se crea una estructura-trama participante, colaborativa. En espacios virtuales esta tarea se hace imprescindible, en cuanto que sobre ella recae no ya la dinámica colaboradora sino la misma dinámica dialógica propia de los encuentros personales. Se cumple así el principio vigotskiano de que la dinámica dialogal se hace posible a través de esa otra de carácter más bien societario o que esta última se actualiza, al tiempo que posibilita, a través del encuentro interpersonal.

En base a las dos reflexiones anteriores, estamos en condiciones de hacer la siguiente consideración a propósito del entorno virtual que viene motivando nuestra reflexión y que recoge otro principio general del enfoque sociocultural en sus diferentes vertientes teóricas. El escenario que ahí se configura no está basado en el funcionamiento del individuo como unidad de análisis y mucho menos pretende simular la mecánica solipsista de la mente humana mediante la creación de espacios, tramas y rutas sometidas a normas y reglas fijas. Frente a ello, se ha creado técnica y pedagógicamente un entorno que se caracteriza básicamente por la apertura de formato y textura así como por funcionar como un sistema cognitivo unificado o, si se prefiere expresarlo en términos vigotskianos, como un sistema funcional.

Detengámonos, aunque sea brevemente, en las características que acabamos de enunciar, comenzando por el final. Ciertamente el entorno virtual que estamos analizando, generado por un determinado diseño técnico, funciona en sentido pedagógico como un sistema funcional de actividad, un grupo de componentes y mecanismos que generan y articulan actividad y orientan la participación vía colaboración; y mientras aquéllos, los componentes y mecanismos, son variables y múltiples, como corresponde a la diversidad de recursos que pueden intervenir y en los que se apoya la generación colectiva de la actividad, el sistema de actividad permanece inalterable.

Quizás se vea con mayor claridad lo que estamos diciendo, si nos fijamos en la dinámica que se activa en algún espacio concreto de nuestro entorno, por ejemplo, en el rotulado "proponer una nueva unidad de formación"; pensemos que el escenario generado en su conjunto no es otra cosa que una especie de trabazón de espacios de este tipo. Pues bien, en este que ahora nos interesa se crea a modo de un sistema cognitivo unificado, donde cada participante actúa en base a las manifestaciones de los demás, sin otro requisito que la participación, que resulta ser así participación guiada. 
Bien merecería esto un análisis más detallado de las distintas formas discursivas de intervención - el papel que desempeñan en la reflexión y en el diálogo los interrogantes, los artificios literarios..., otros muchos dispositivos dialogísticos..., el intercambio simulado y permanente de funciones, el esfuerzo público por recuperar la información contextual...- así como de otro tipo de recursos, simbólicos y lingüísticos, que aparecen en relaciones mediadas con dispositivos tecnológicos y que en el fondo constituye todo ello, en expresión vigotskiana, distintas formas de andamiar y articular la intersubjetividad. Esta reflexión nos introduce en esa otra línea de investigación de los entornos virtuales de aprendizaje que más arriba entendíamos como complementaria, la perspectiva semiótica.

Si ahora volvemos a retomar la visión global del entorno, entendemos más fácilmente lo que anteriormente decíamos, que el entorno virtual objeto de nuestro análisis es un sistema funcional de actividad en cuanto que está compuesto por varios y diversos espacios del tipo del que acabamos de analizar, espacios recursivos en su interior, que van generando con participación colectiva progreso en forma elíptica, y entrelazados de forma que se teje la cadena colaborativa; esta disposición estructural también puede ser vista como otra forma de andamiar el escenario para desplegar intersubjetividad.

La otra característica a la que hacíamos referencia más arriba era el carácter abierto del entorno, tanto si lo consideramos en su conjunto como en sus componentes. La intersubjetividad no requiere de cierres prematuros basados en intervenciones personalistas, estáticas y definitivamente dadas; esto es más propio de los sistemas de aprendizaje que subyacen en la metáfora del ordenador como tutor y que se apoyan en una interpretación de la inteligencia como computación de normas y reglas fijas. Frente a ello, el entorno que estamos caracterizando pedagógicamente se sustenta en una concepción de la inteligencia de tipo adaptativo, más que en una concepción de la inteligencia formal, abstracta, representacional y computacional, que terminan proporcionando al entorno una imagen de mecanicismo, linealidad y constricción de la intersubjetividad.

Esta reflexión que acabamos de hacer a propósito del carácter abierto de nuestro entorno, tanto globalmente considerado como en sus componentes, despierta un tema de indudable interés para todo software educativo que pretenda promover la colaboración; nos estamos refiriendo ahora a la conveniencia de introducir algún artificio que se encargue de gestionar el tipo de participación de los intervinientes, o si se prefiere, la aportación y tipo de contribución de los participantes, en definitiva, lo que podríamos llamar un gestor de conocimiento.

Cierto es que la estructura que hemos proporcionado a los componentes y funcionamiento de nuestro entorno virtual no es otra cosa que formas artificiales de gestionar la presencia y participación del interviniente, pero en alguna ocasión se ha mostrado insuficiente. Con alguna frecuencia nos hemos encontrado que la intervención de un participante se acumulaba temáticamente en uno de los espacios o nichos del entorno, por ejemplo, en una de las tareas a realizar el participante vertía todas sus opiniones sobre el tema con independencia de que algunas 
de ellas correspondieran en su totalidad o en parte a otras tareas, que recogían otras vertientes de análisis y tratamiento del tema; es ésta una forma de reflexionar por cúmulos, por montones de cosas a decir en una misma intervención, trasladando quizás el modelo de funcionamiento de nuestras intervenciones en contextos presenciales a los ámbitos virtuales, de manera que disminuye y se bloquea la reflexión conjunta en la medida en que no se distribuyen las aportaciones individuales, disminuyendo a su vez las posibilidades de contraste de reflexiones entre los sujetos. De alguna manera, el incremento de tareas o actividades a realizar, al que nos hemos referido anteriormente, contrarrestaba este problema que genera la reflexión por amontonamiento y que suele terminar en un intercambio de monólogos que en nada favorecen la intersubjetividad.

Decíamos, en un intento por explicar este comportamiento verdaderamente perjudicial para la actividad colectiva, que los participantes que actuaban así estaban trasladando a los espacios virtuales esquemas de funcionamiento propios de los contextos presenciales, olvidando que la acumulación de reflexión o conocimiento en un solo espacio en contextos virtuales hace desaparecer, si no se toman precauciones al respecto, la variable relativa al momento de realización de la intervención y, por ende, a su contexto de referencia. Queremos decir con ello que, mientras en contextos presenciales podemos acumular en una misma intervención varias reflexiones referidas a distintos aspectos, correlativas a su vez con reflexiones de otros participantes, para lo que utilizamos para su/nuestra exposición y ordenación mental variables como el momento en que se produjeron esas intervenciones, el contexto en que se realizaron, incluso la ubicación física de quien las hizo..., en los escenarios virtuales la presencia de unas variables inhibe la actuación de otras; en el caso concreto que estamos comentando la variable espacio interfiere la dimensión temporal de la intervención hasta el punto de simular cuasi simultaneidad y, por analogía plástica, provoca amontonamiento de la reflexión.

Este comportamiento se vuelve contra el principio de la búsqueda de las máximas facilidades y oportunidades para la acción de una mente, una cognición y un aprendizaje distribuido, contra el principio de la máxima distribución de nuestra mente y de nuestra actividad, principio del que partíamos en el inicio de nuestra reflexión y que se ha revelado como el leit motiv de la caracterización pedagógica de los entornos virtuales, en cuanto que constituye un potente mecanismo favorecedor de la dinámica de la intersubjetividad. Contar en los espacios virtuales con mecanismos de distribución del conocimiento significa incrementar las oportunidades de intervención de los participantes, favorecer los contrastes de opinión y aportaciones y, en última instancia, orientar la actividad en una dirección colaborativa ${ }^{16}$.

16. Las formas como distribuir el conocimiento pueden ser muchas, como ya hemos señalado, pero ahora queremos añadir una que requiere de un mayor análisis a propósito de su viabilidad técnica: la introducción de mecanismos que generen el autodesarrollo, la autoexpansión, la replicación de partes y/o funciones del propio entorno en sentido técnico; se lograría así el auténtico carácter abierto que ha guiado esta larga reflexión. 


\section{CONCLUSIÓN: RECUPERANDO Y ABRIENDO PERSPECTIVAS}

Desde la consideración de las nuevas tecnologías no como meros artefactos o instrumentos al servicio de un contexto y enfoque tradicional de la educación sino como artificios capaces de generar nuevos contextos y escenarios que posibilitan acciones educativas, hemos llegado a la determinación de algunos principios fundamentales que permiten configurar con intención educativa esos entornos virtuales, pasando por distintas corrientes teóricas ancladas en el enfoque sociocultural como marco de inteligibilidad pedagógica de los procesos y acciones que en esos entornos se producen.

Además de los tres o cuatro principios que hemos identificado, podríamos comentar otros muchos aspectos sobre la forma de diseñar y estructurar técnica y pedagógicamente esos entornos de manera que sirvan de catalizadores de los procesos que se consideran imprescindibles para que la actividad educativa tenga lugar. En cualquier caso, parece obvio que, mientras no consigamos clarificar ésta y otras cuestiones, poniendo de relieve las características o vectores necesarios que deben caracterizar la relación de educadores y educandos en los espacios virtuales, seguiremos teniendo espacios anónimos, procesos descafeinados y resultados educativos carentes de significación y rigor pedagógico.

Hoy por hoy no toda oferta autodenominada espacio virtual de formación es válida pedagógicamente hablando. En líneas generales y sin entrar en debates particulares, no debemos confundir lo que es la mera información sin más con aquellos otros espacios que además van acompañados de diseños y procesos de comunicación, de formación, de educación. Sólo si tenemos en cuenta estos aspectos específicamente educativos, podremos hablar de espacios virtuales como auténticos ámbitos de comunicación, interacción y actividad social, de información, creación y distribución de conocimiento, y en conclusión, de educación y de formación.

Urge en este sentido diferenciar entre mecanismos, diseños o herramientas de propaganda, editoriales encubiertas, donde las tecnologías de la información y la comunicación son auténticas herramientas de información y administración de información, pero que quedan muy lejos de ser considerados centros virtuales de formación. Ésta es quizás una de las líneas de investigación más prioritarias; urge tener criterios que permitan discernir en torno a la validez de todos aquellos entornos educativos que dicen ser gestores de educación y formación y que, en algunos casos, no van más allá de ser administradores de información. 


\section{BIBLIOGRAFIA}

BATEs, A. (1995) Technology, open learning and distance educatio. York, Routledge.

BOSCO, J. (1995) Schooling and Learning in an Information Society, en UNITED STATES CONGRESS. Education and Technology: Future Visions. Washington, Government Printing Office.

Brown, J. S.; Collins, A. \& Duglid, P. (1989) Situated cognition and the culture of learning, Educational Researcher, 18, 32-42.

ClANCEY, W. J. (1999) Situated Cognition. Cambridge, Cambridge University Press.

Cole, M. y ENGeström, Y. (2001) Enfoque histórico-cultural de la cognición distribuida, en Salomon, G. (comp.) Cogniciones distribuidas. Consideraciones psicológicas y educativas. Buenos Aires, Amorrortu, 23-74.

Crook, Ch. (1998) Ordenadores y aprendizaje colaborativo. Madrid, Ministerio de Educación y Cultura-Ediciones Morata.

Dillenibourg, P. (ed.) (1999) Collaborative-learning: Cognitive and computational approaches. Oxford, Elsevier.

Dillenbourg, P. y Self, A. (1992) A computacional approach to socially distributed cognition, European Journal of Psichology of Education, VII, 4, 352-373.

ENGESTRÖM, Y. (1987) Learning by Expanding: An activity-theoretical approach to developmental research. Helsinki, Orienta-Konsultut.

ENGESTRÖM, Y. y MidDleton, D. (1996) Cognition and communication at work. Cambridge, Cambridge University Press.

GerRY, S. (ed.) (2002) Proceedings of CSCL 2002. New Jersey, Lawrence Erlbaum Associates, Inc. Hillsdale.

Harnad, S. (1991) Post-Guttemberg Galaxy: The Fourth Revolution in the Means of Production of Knowledge, The Public-Acces Computer System Review, 21 (1), 39-53.

Hutchins, E. (1995) Cognition in the wild. Cambridge, MA, MIT Press.

Johnson, D. W. y Johnson, R. T. (1994) Structuring academic controversy, en Sharan, S. (ed.). Handbook of cooperative learning methods. Westport, Praeger.

Johnson, D.; Johnson, R. y HolubeC, E. (1999) El aprendizaje cooperativo en el aula. Buenos Aires, Paidós.

Kolb, D. A. (1998) Experiential learning: From discourse model to conversation. Lifelong Learning in Europe, 3, 148-153.

Koschmann, T. (1996) CSCL, Theory and Practice of an emerging paradigm. New Jersey, Lawrence Erlbaum Associates.

LABBO, L. D. (2001) Toward a Vision of the Future Role of Technology in Literacy Education, en VV.AA. Literacy for the $21^{\text {st }}$ century: Technological transformation in a post-typograpbical world. New York, Erlbaum.

LAVE, J. (1991) La cognición en la práctica. Barcelona, Paidós.

- (2001) La práctica del aprendizaje, en Chaikun, S. y LAve, J. Estudiar las prácticas. Perspectivas sobre actividad y contexto. Buenos Aires, Amorrortu.

LAVE, J. y WeNGER, E. (1991). Situated Learning. Legitimate perpherical participation. Cambridge, Cambrictge University Press.

Levinson, P. (1990) Computer Conferencing in the Context of the Evolutions of Media, en Harasim, L. M. Online Education. Perspectives on a New Environment. New York, Praeger Press, 3-14. 
LEWIS, R. (2001) Grupos de trabajo en comunidades virtuales, Jornadas de la red FREREF NTIC, http://www.uoc.edu/web/esp/art/uoc/lewis0102/lewis0102.html

LOzARES, C. (2000) La actividad situada y/o el conocimiento socialmente distribuido. Papers, Revista de Sociología, 62, 97-131.

Mclellan, H. (1995) Situated Learning Perspectives. New Jersey, Educational Technology Publications.

Newman, D.; Griffin, P. y Cole, F. (1991) La zona de construcción del conocimiento. Madrid, Morata.

Norman, D. A. (1993) Cognition in the Head and in the World: An Introduction to the Special Issue on Situated Action, Cognitive Science, 17, 1-6.

Olson, D. R. y Torrance, N. (comp.) (1991) Cultura escrita y oralidad. Barcelona, Gedisa.

ONG, W. J. (1995) Orality \& Literacy: The Technologizing of the World. London, Routledge.

PAPERT, S. (1993) The Children's Machine: Rethinking School in the Age of the Computer. New York, Basic Books.

PEA, R. D. (1993) Prácticas de inteligencia distribuida y diseños para la educación, en SAloMON, G. (comp.) (2001) Cogniciones distribuidas. Consideraciones psicológicas y educativas. Buenos Aires, Amorrortu, 75-125.

Salomon, G. (2001) No hay distribución sin la cognición de los individuos: un enfoque interactivo dinámico, en Salomon, G. (comp.). Cogniciones distribuidas. Consideraciones psicológicas y educativas. Buenos Aires, Amorrortu, 153-184.

Suchman, L. (1987) Plans and situated action: the problem of humanmachine communication. Cambridge, Cambridge University Press.

- (1993) Respuesta a la acción situada de Vera y Simon: una intepretacion simbólica, Cognitive Science, 17, 71-75.

VAN JOOLINGEN, W. R. (2000) Designing for collaborative discovery learning, en GAUTHIER, G.; Frasson, C. \& VANIEHN, K. (eds.). Proceedings of 5th International Conference on Intelligent Tutoring Systems. Berlin, Heidelberg, Springer, 202-211.

WALDEGg CASANOVA, G. (2002) El uso de las nuevas tecnologías para la enseñanza y el aprendizaje de las ciencias, Revista Electrónica de Investigación Educativa, 4, 1.

Wenger, E. (2001) Comunidades de práctica. Aprendizaje, significado e identidad. Barcelona, Paidós. 